\title{
Strømgren photometry of SX Phe = HD 223065
}

\author{
A. Stankov ${ }^{1}$, D. Sinachopoulos ${ }^{2}$, E. Elst ${ }^{3}$, M. Breger ${ }^{1}$ \\ ${ }^{1}$ Institut für Astronomie, Türkenschanzstrasse 17, 1180 Vienna, Austria \\ 2 Institute of Astronomy and Astrophysics, National Observatory of Athens, I. \\ Metaxa and Bas. Pavlou, 15236 Athens, Greece \\ ${ }^{3}$ Koninklijke Sterrenwacht van België, Ringlaan 3, 1180 Uccle, Belgium
}

\begin{abstract}
We present the analysis of more than 220 photometric measurements of the variable star SX Phe. The observations were made with the Johnson $V$ and the four Strømgren $(u, v, b, y)$ filters. An analysis of the parameters $\delta c_{1}$ and $\delta m_{1}$ is presented. The behavior of $\delta c_{1}$ during the luminosity variations of the star corresponds to that expected from the standard luminosity calibrations by Crawford (1979) with $\delta V=-10 \delta c_{1}$. The behavior of the metallicity index, $\delta m_{1}$, does not correspond to the expectations from the standard calibrations.
\end{abstract}

\section{Introduction}

SX Phe stars are a Pop. II subgroup of the $\delta$ Scuti stars. In order to shed light on the variability of SX Phe and related stars, many studies of SX Phe have been undertaken since the report on our previous observations (Elst 1978). Rodriguez et al. (1990) used Strømgren photometry to study the behavior of the $\delta m_{1}$ index for SX Phe stars. In addition, Garrido et al. (1990) used uvby photometry for the identification of pulsation modes (modal discrimination) of the pulsating $\delta$ Scuti stars. The observations presented here were obtained to provide deeper understanding of the variability of SX Phe in the Strømgren filters.

\section{Observations and data reduction}

The ESO $50 \mathrm{~cm}$ telescope was used to monitor SX Phe during the nights of 1992 October 18 and 31, as well as 1992 November 1. HD 223011 was used as 

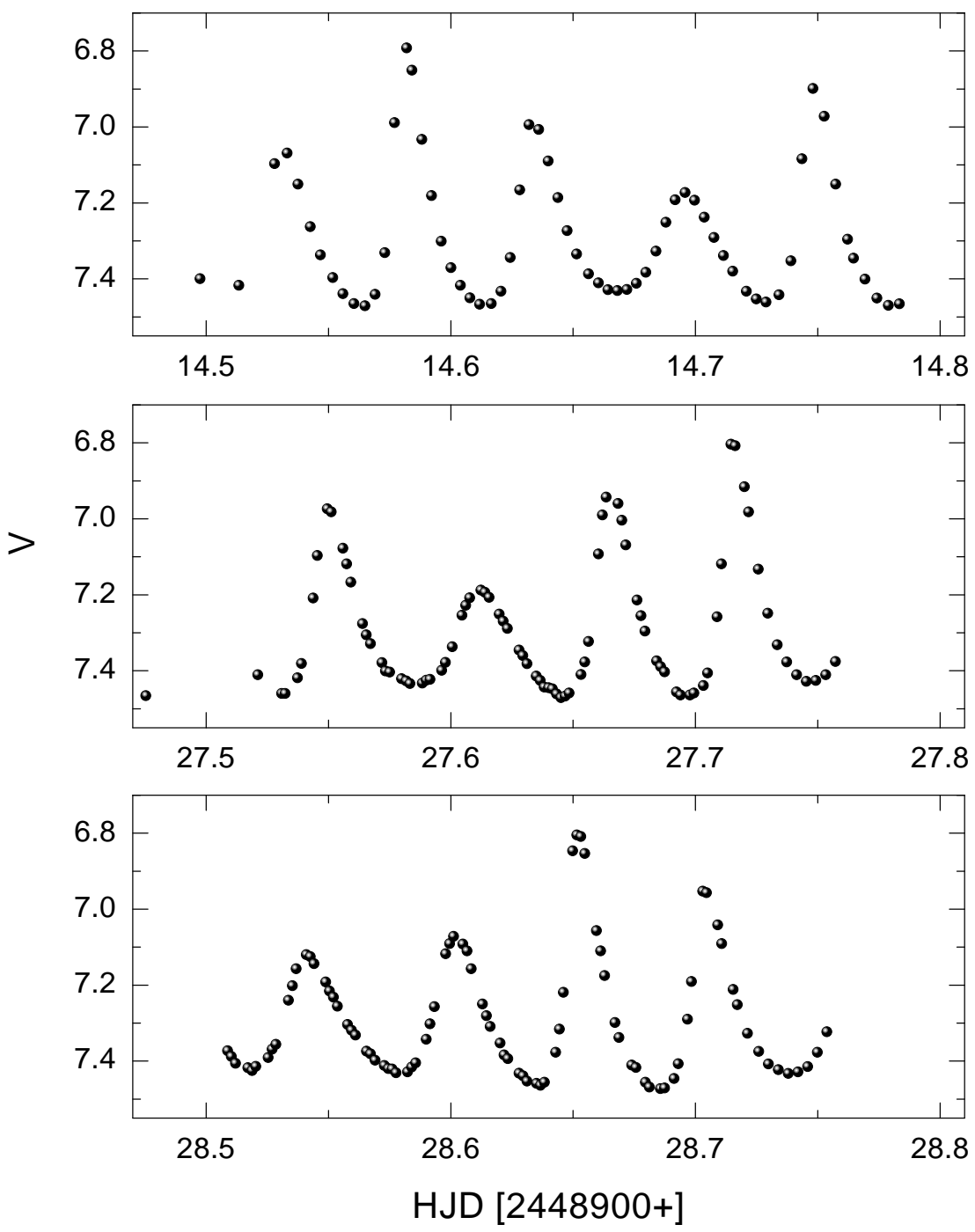

Figure 1: Photometry of SX Phe in $V$

a comparison star. The photometer of the $50 \mathrm{~cm}$ ESO telescope is only a singlechannel photometer. Consequently, the measurements through the four filters were not obtained simultaneously, as was the case in Garrido et al. (1990). Due to technical problems, we had to use two different photomultipliers for our 
observations. During the first night we used a HAMAMATSU and during the other two nights a EMI 9789QB tube.

In addition, we observed 16 bright photometric standard stars each night for transforming our observations into the standard uvby system. The SNOPY software on theHP 1000 computer was used for this transformation. The comparison star did show any significant variability during this period. From the statistical fluctuations of its reduced magnitudes and indices we estimate that the accuracy of a single HD 223011 measurement is $\sigma_{V}=0.006, \sigma_{(b-y)}=$ $0.005, \sigma_{m_{1}}=0.008, \sigma_{c_{1}}=0.007 \mathrm{mag}$.
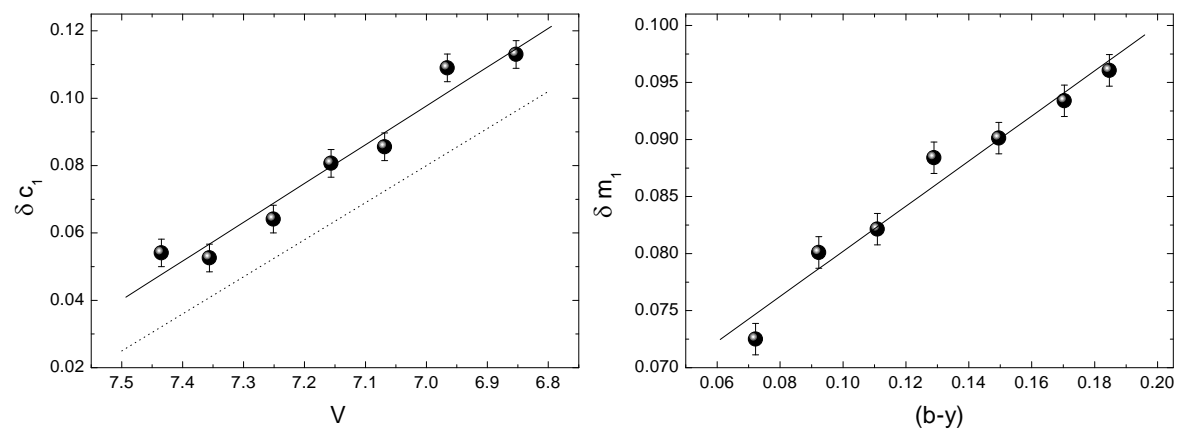

Figure 2: Left pannel: $\delta c_{1}$ versus $V$; dotted line: expected slope from calibrations from Crawford. Right pannel: $\delta m_{1}$ versus $(b-y)$.

Since SX Phe is about 0.9 magnitudes fainter than the comparison star, we expect that the accuracy of measurement is lower for SX Phe. More than 10000 counts per exposure (30 seconds each) of SX Phe were collected in each filter during the first night using the HAMAMATSU photo-multiplier. The efficiency of the EMI 9789QB tube was somewhat lower.

Table 1 lists the measurements of SX Phe, while Figure 1 shows the light curves of SX Phe obtained during three nights.

\section{Variations of the Stromgren parameters during the pulsational cycles}

Crawford (1979) has presented calibrations of these parameters, based on a large number of different stars. A pulsating star such as SX Phe changes its luminosity and temperature during its pulsation cycle, but is not affected by a variable abundance. This provides an excellent opportunity to check these calibrations whether also apply the atmospheric changes during pulsation. 
We can now turn to the variations of the metallicity parameter for $A / F$ stars, $\delta m_{1}$, and the luminosity parameter, $\delta c_{1}$. The observed variations are shown in Fig. 2. For $\delta c_{1}$, we derive a slope of $\delta V / \delta c_{1}=9.8$, in good agreement with the expected value of 9.0 derived for different stars by Crawford. The two slopes are also shown in Fig. 2, where the Crawford slope is displaced vertically for greater clarity.

The right panel of Fig. 2 shows the relation between $\delta m_{1}$ and color, $b-y$. A definite non-zero slope is found. A comparison with the standard values given by Crawford (1979), describing the variation of $\delta m_{1}$ with temperature, shows very poor agreement. A logical, but incorrect, interpretation would be that the metal content changes during the cycle! We note here that SX Phe is extremely metal-poor and that the calibrations were set up for stars with near-solar abundances. The calibrations, therefore, cannot be expected to hold for SX Phe.

The behavior of SX Phe confirms the observed behavior of two other (metalpoor) SX Phe stars, KZ Hya and CY Aqr (Rodriguez et al. 1990).

\section{References}

Crawford, D. L. 1979, AJ 84, 1858

Elst E. 1978, A\&AS 32, 161

Garrido, R., Garcia-Lobo, E., Rodriguez, E. 1990, A\&A 234, 262

Rodriguez, E., Rolland, A., Lopez de Coca, R., et al. 1990, Rev. Mexic.

Astron. Astrof. 21, 386 
Table 1: Measurements of SX Phe

\begin{tabular}{||r|c|c|c|c||}
\hline HJD +2448900 & $\mathrm{~V}$ & $(\mathrm{~b}-\mathrm{y})$ & $\mathrm{m}_{1}$ & $\mathrm{c}_{1}$ \\
\hline 14.4976 & 7.400 & .185 & .076 & .781 \\
14.5134 & 7.417 & .163 & .096 & .763 \\
14.5280 & 7.097 & .097 & .123 & .920 \\
14.5332 & 7.069 & .108 & .123 & .938 \\
14.5376 & 7.151 & .122 & .126 & .900 \\
& & & & \\
14.5426 & 7.263 & .152 & .098 & .863 \\
14.5468 & 7.337 & .162 & .097 & .821 \\
14.5518 & 7.397 & .179 & .086 & .793 \\
14.5560 & 7.439 & .181 & .089 & .771 \\
14.5605 & 7.465 & .187 & .082 & .760 \\
& & & & \\
14.5650 & 7.471 & .182 & .080 & .758 \\
14.5691 & 7.441 & .174 & .081 & .750 \\
14.5731 & 7.331 & .135 & .093 & .786 \\
14.5771 & 6.989 & .022 & .138 & .948 \\
14.5820 & 6.792 & .057 & .138 & 1.036 \\
& & & & \\
14.5842 & 6.851 & .074 & .138 & 1.037 \\
14.5882 & 7.033 & .114 & .126 & .963 \\
14.5922 & 7.181 & .140 & .109 & .905 \\
14.5962 & 7.301 & .161 & .096 & .851 \\
14.6001 & 7.371 & .175 & .082 & .821 \\
& & & & \\
14.6040 & 7.417 & .182 & .084 & .792 \\
14.6079 & 7.450 & .188 & .080 & .773 \\
14.6118 & 7.467 & .188 & .077 & .767 \\
14.6166 & 7.465 & .180 & .088 & .745 \\
14.6206 & 7.433 & .168 & .085 & .757 \\
& & & & \\
14.6244 & 7.344 & .143 & .107 & .777 \\
14.6282 & 7.166 & .087 & .118 & .882 \\
14.6320 & 6.994 & .075 & .137 & .968 \\
14.6360 & 7.007 & .095 & .126 & .977 \\
& & continued on next page \\
\hline & & & \\
& & & \\
& & & \\
140 &
\end{tabular}




\begin{tabular}{|c|c|c|c|c|}
\hline \multicolumn{5}{|c|}{ continued from previous page } \\
\hline HJD +2448900 & $\mathrm{V}$ & $(b-y)$ & $\mathrm{m}_{1}$ & $\mathrm{C}_{1}$ \\
\hline 14.6399 & 7.090 & .114 & .123 & .947 \\
\hline 14.6438 & 7.186 & .139 & .125 & .892 \\
\hline 14.6476 & 7.273 & .155 & .115 & .854 \\
\hline 14.6515 & 7.335 & .163 & .116 & .823 \\
\hline 14.6564 & 7.387 & .173 & .102 & .809 \\
\hline 14.6604 & 7.411 & .183 & .096 & .783 \\
\hline 14.6643 & 7.429 & .182 & .092 & .781 \\
\hline 14.6682 & 7.431 & .175 & .111 & .754 \\
\hline 14.6720 & 7.428 & .174 & .105 & .759 \\
\hline 14.6759 & 7.412 & .168 & .107 & .761 \\
\hline 14.6798 & 7.383 & .157 & .114 & .780 \\
\hline 14.6839 & 7.327 & .144 & .115 & .815 \\
\hline 14.6880 & 7.251 & .120 & .130 & .857 \\
\hline 14.6918 & 7.192 & .116 & .131 & .890 \\
\hline 14.6958 & 7.173 & .121 & .127 & .903 \\
\hline 14.6997 & 7.193 & .136 & .115 & .898 \\
\hline 14.7037 & 7.238 & .141 & .126 & .857 \\
\hline 14.7076 & 7.291 & .156 & .115 & .835 \\
\hline 14.7115 & 7.339 & .166 & .109 & .817 \\
\hline 14.7153 & 7.380 & .172 & .106 & .800 \\
\hline 14.7209 & 7.433 & .180 & .098 & .783 \\
\hline 14.7249 & 7.453 & .182 & .098 & .776 \\
\hline 14.7289 & 7.461 & .181 & .101 & .757 \\
\hline 14.7342 & 7.442 & .171 & .098 & .764 \\
\hline 14.7391 & 7.353 & .135 & .113 & .789 \\
\hline 14.7436 & 7.084 & .068 & .134 & .925 \\
\hline 14.7481 & 6.899 & .071 & .140 & 1.012 \\
\hline 14.7527 & 6.972 & .100 & .140 & .998 \\
\hline 14.7574 & 7.151 & .135 & .136 & .905 \\
\hline 14.7622 & 7.296 & .161 & .107 & .860 \\
\hline & & ntinu & on $n$ & xt page \\
\hline
\end{tabular}




\begin{tabular}{|c|c|c|c|c|}
\hline \multicolumn{5}{|c|}{ continued from previous page } \\
\hline HJD +2448900 & $\mathrm{V}$ & $(b-y)$ & $\mathrm{m}_{1}$ & $\mathrm{c}_{1}$ \\
\hline 14.7647 & 7.346 & .171 & .101 & .829 \\
\hline 14.7694 & 7.401 & .183 & .091 & .812 \\
\hline 14.7742 & 7.451 & .186 & .105 & .771 \\
\hline 14.7789 & 7.470 & .191 & .094 & .758 \\
\hline 14.7834 & 7.466 & .184 & .088 & .763 \\
\hline 27.5212 & 7.411 & .200 & .085 & .783 \\
\hline 27.5309 & 7.460 & .197 & .084 & .765 \\
\hline 27.5324 & 7.460 & .190 & .092 & .757 \\
\hline 27.5374 & 7.419 & .166 & .103 & .753 \\
\hline 27.5390 & 7.381 & .166 & .091 & .771 \\
\hline 27.5438 & 7.209 & .101 & .117 & .868 \\
\hline 27.5455 & 7.097 & .072 & .130 & .936 \\
\hline 27.5496 & 6.974 & .085 & .132 & .999 \\
\hline 27.5512 & 6.982 & .095 & .128 & 1.002 \\
\hline 27.5560 & 7.078 & .121 & .126 & .959 \\
\hline 27.5575 & 7.119 & .128 & .127 & .934 \\
\hline 27.5592 & 7.167 & .136 & .119 & .920 \\
\hline 27.5640 & 7.276 & .154 & .117 & .868 \\
\hline 27.5655 & 7.306 & .159 & .114 & .855 \\
\hline 27.5672 & 7.329 & .164 & .117 & .832 \\
\hline 27.5719 & 7.379 & .171 & .117 & .800 \\
\hline 27.5734 & 7.401 & .168 & .112 & .801 \\
\hline 27.5751 & 7.404 & .179 & .104 & .794 \\
\hline 27.5799 & 7.421 & .191 & .093 & .782 \\
\hline 27.5818 & 7.427 & .181 & .106 & .771 \\
\hline 27.5834 & 7.434 & .177 & .113 & .755 \\
\hline 27.5884 & 7.433 & .171 & .109 & .775 \\
\hline 27.5900 & 7.425 & .174 & .106 & .770 \\
\hline 27.5916 & 7.423 & .169 & .111 & .762 \\
\hline 27.5963 & 7.399 & .156 & .116 & .775 \\
\hline 27.5979 & 7.378 & .146 & .126 & .787 \\
\hline
\end{tabular}




\begin{tabular}{|c|c|c|c|c|}
\hline \multicolumn{5}{|c|}{ continued from previous page } \\
\hline HJD +2448900 & $\mathrm{V}$ & $(b-y)$ & $\mathrm{m}_{1}$ & $\mathrm{c}_{1}$ \\
\hline 27.6007 & 7.337 & .134 & .129 & .818 \\
\hline 27.6046 & 7.254 & .121 & .133 & .859 \\
\hline 27.6062 & 7.228 & .119 & .132 & .877 \\
\hline 27.6078 & 7.208 & .119 & .134 & .886 \\
\hline 27.6124 & 7.188 & .125 & .130 & .895 \\
\hline 27.6140 & 7.194 & .133 & .120 & .906 \\
\hline 27.6157 & 7.207 & .131 & .134 & .881 \\
\hline 27.6199 & 7.251 & .142 & .121 & .877 \\
\hline 27.6215 & 7.270 & .143 & .127 & .864 \\
\hline 27.6232 & 7.289 & .155 & .119 & .850 \\
\hline 27.6280 & 7.346 & .164 & .106 & .825 \\
\hline 27.6295 & 7.360 & .170 & .104 & .811 \\
\hline 27.6312 & 7.382 & .169 & .105 & .810 \\
\hline 27.6350 & 7.414 & .173 & .110 & .783 \\
\hline 27.6366 & 7.426 & .181 & .103 & .773 \\
\hline 27.6382 & 7.443 & .180 & .103 & .768 \\
\hline 27.6400 & 7.445 & .180 & .107 & .771 \\
\hline 27.6416 & 7.448 & .189 & .095 & .765 \\
\hline 27.6433 & 7.461 & .180 & .103 & .759 \\
\hline 27.6451 & 7.471 & .174 & .105 & .766 \\
\hline 27.6468 & 7.467 & .175 & .112 & .741 \\
\hline 27.6484 & 7.459 & .178 & .101 & .759 \\
\hline 27.6533 & 7.410 & .155 & .105 & .783 \\
\hline 27.6548 & 7.377 & .134 & .126 & .772 \\
\hline 27.6564 & 7.323 & .127 & .118 & .801 \\
\hline 27.6604 & 7.093 & .079 & .114 & .935 \\
\hline 27.6620 & 6.990 & .061 & .138 & .970 \\
\hline 27.6636 & 6.943 & .065 & .136 & 1.001 \\
\hline 27.6684 & 6.960 & .089 & .139 & 1.005 \\
\hline 27.6700 & 7.004 & .107 & .130 & .991 \\
\hline 27.6716 & 7.069 & .111 & .141 & .945 \\
\hline \multicolumn{5}{|c|}{ continued on next page } \\
\hline
\end{tabular}




\begin{tabular}{|c|c|c|c|c|}
\hline \multicolumn{5}{|c|}{ continued from previous page } \\
\hline HJD +2448900 & $\mathrm{V}$ & $(b-y)$ & $\mathrm{m}_{1}$ & $c_{1}$ \\
\hline 27.6762 & 7.214 & .151 & .112 & .894 \\
\hline 27.6778 & 7.255 & 155 & .121 & .863 \\
\hline 27.6794 & 7.296 & .160 & .120 & .836 \\
\hline 27.6842 & 7.374 & .178 & .105 & .808 \\
\hline 27.6858 & 7.389 & 183 & .099 & .806 \\
\hline 27.6874 & 7.403 & .188 & .103 & .787 \\
\hline 27.6923 & 7.456 & .188 & .094 & .784 \\
\hline 27.6939 & 7.464 & 188 & .104 & .760 \\
\hline 27.6978 & 7.464 & 193 & .091 & .758 \\
\hline 27.6994 & 7.459 & 192 & .088 & .765 \\
\hline 27.7033 & 7.439 & 169 & .099 & .759 \\
\hline 27.7050 & 7.406 & 164 & .105 & .755 \\
\hline 27.7089 & 7.258 & .120 & 109 & .807 \\
\hline 27.7107 & 7.119 & .068 & .113 & .895 \\
\hline 27.7146 & 6.804 & .051 & 143 & 1.021 \\
\hline 27.7163 & 6.808 & .065 & 139 & 1.035 \\
\hline 27.7201 & 6.916 & .094 & .141 & 1.022 \\
\hline 27.7218 & 6.982 & .118 & .132 & .978 \\
\hline 27.7257 & 7.133 & 142 & .115 & .937 \\
\hline 27.7296 & 7.249 & .156 & .113 & .869 \\
\hline 27.7335 & 7.332 & .173 & .104 & .823 \\
\hline 27.7374 & 7.377 & .185 & .097 & .811 \\
\hline 27.7414 & 7.411 & .189 & .094 & .773 \\
\hline 27.7454 & 7.428 & 190 & .099 & .759 \\
\hline 27.7493 & 7.426 & 191 & .085 & .767 \\
\hline 27.7533 & 7.411 & 183 & .089 & .761 \\
\hline 27.7573 & 7.376 & 167 & .106 & .757 \\
\hline 28.5088 & 7.373 & .181 & .109 & .769 \\
\hline 28.5104 & 7.388 & .190 & .094 & .774 \\
\hline 28.5121 & 7.406 & .181 & .106 & .755 \\
\hline 28.5171 & 7.418 & .193 & .096 & .733 \\
\hline
\end{tabular}




\begin{tabular}{|c|c|c|c|c|}
\hline \multicolumn{5}{|c|}{ continued from previous page } \\
\hline HJD +2448900 & $\mathrm{V}$ & $(b-y)$ & $\mathrm{m}_{1}$ & $\mathrm{c}_{1}$ \\
\hline 28.5188 & 7.425 & .186 & .094 & .742 \\
\hline 28.5204 & 7.414 & .189 & .096 & .724 \\
\hline 28.5254 & 7.391 & .169 & .099 & .747 \\
\hline 28.5270 & 7.369 & .182 & .078 & .761 \\
\hline 28.5286 & 7.356 & .157 & .101 & .765 \\
\hline 28.5337 & 7.240 & .132 & .098 & .845 \\
\hline 28.5353 & 7.202 & .111 & .118 & .869 \\
\hline 28.5369 & 7.157 & .118 & .105 & .902 \\
\hline 28.5410 & 7.120 & .125 & .102 & .930 \\
\hline 28.5426 & 7.125 & .122 & .110 & .922 \\
\hline 28.5442 & 7.144 & .116 & .126 & .897 \\
\hline 28.5489 & 7.192 & .136 & .111 & .886 \\
\hline 28.5505 & 7.216 & .141 & .109 & .881 \\
\hline 28.5521 & 7.232 & .149 & .106 & .860 \\
\hline 28.5537 & 7.256 & .151 & .107 & .843 \\
\hline 28.5579 & 7.304 & .163 & .102 & .813 \\
\hline 28.5595 & 7.319 & .167 & .100 & .805 \\
\hline 28.5611 & 7.332 & .173 & .094 & .801 \\
\hline 28.5656 & 7.374 & .174 & .096 & .785 \\
\hline 28.5672 & 7.381 & .178 & .099 & .765 \\
\hline 28.5691 & 7.398 & .180 & .088 & .782 \\
\hline 28.5729 & 7.412 & .184 & .087 & .774 \\
\hline 28.5745 & 7.420 & .177 & .097 & .760 \\
\hline 28.5761 & 7.421 & .179 & .099 & .750 \\
\hline 28.5777 & 7.431 & .176 & .095 & .756 \\
\hline 28.5824 & 7.429 & .168 & .094 & .763 \\
\hline 28.5841 & 7.416 & .173 & .090 & .759 \\
\hline 28.5857 & 7.405 & .163 & .099 & .761 \\
\hline 28.5900 & 7.343 & .146 & .098 & .791 \\
\hline 28.5916 & 7.302 & .138 & .100 & .810 \\
\hline 28.5934 & 7.257 & .122 & .109 & .837 \\
\hline \multicolumn{5}{|c|}{ continued on next page } \\
\hline
\end{tabular}




\begin{tabular}{|c|c|c|c|c|}
\hline \multicolumn{5}{|c|}{ continued from previous page } \\
\hline HJD +2448900 & $\mathrm{V}$ & $(b-y)$ & $\mathrm{m}_{1}$ & $\mathrm{C}_{1}$ \\
\hline 28.5980 & 7.118 & .100 & .118 & .913 \\
\hline 28.5996 & 7.091 & .095 & .122 & .927 \\
\hline 28.6012 & 7.072 & .099 & .121 & .939 \\
\hline 28.6050 & 7.092 & .108 & .125 & .933 \\
\hline 28.6067 & 7.110 & .124 & .118 & .915 \\
\hline 28.6084 & 7.157 & .117 & .125 & .911 \\
\hline 28.6130 & 7.250 & 149 & .099 & .857 \\
\hline 28.6146 & 7.281 & 150 & .108 & .823 \\
\hline 28.6162 & 7.309 & .158 & .094 & .826 \\
\hline 28.6202 & 7.353 & .175 & .090 & .789 \\
\hline 28.6218 & 7.384 & .165 & .095 & .797 \\
\hline 28.6234 & 7.394 & .175 & .089 & .785 \\
\hline 28.6280 & 7.432 & .185 & .074 & .778 \\
\hline 28.6296 & 7.439 & .189 & .075 & .763 \\
\hline 28.6312 & 7.453 & .182 & .079 & .756 \\
\hline 28.6351 & 7.459 & .170 & .089 & .744 \\
\hline 28.6367 & 7.464 & .175 & .096 & .736 \\
\hline 28.6383 & 7.456 & .172 & .087 & .750 \\
\hline 28.6429 & 7.377 & .144 & .099 & .753 \\
\hline 28.6445 & 7.316 & .125 & .097 & .791 \\
\hline 28.6461 & 7.219 & .087 & .107 & .839 \\
\hline 28.6499 & 6.847 & .039 & .126 & 1.005 \\
\hline 28.6516 & 6.805 & .051 & .120 & 1.042 \\
\hline 28.6532 & 6.809 & .066 & .116 & 1.054 \\
\hline 28.6548 & 6.854 & .065 & .137 & 1.040 \\
\hline 28.6596 & 7.057 & .117 & .117 & .959 \\
\hline 28.6613 & 7.110 & .134 & .111 & .929 \\
\hline 28.6629 & 7.175 & .134 & .115 & .897 \\
\hline 28.6672 & 7.299 & .164 & .099 & .838 \\
\hline 28.6688 & 7.338 & .167 & .094 & .827 \\
\hline 28.6740 & 7.411 & .176 & .083 & .810 \\
\hline
\end{tabular}




\begin{tabular}{||r|c|c|c|c||}
\hline \multicolumn{6}{|c||}{ continued from previous page } \\
\hline $\mathrm{HJD}+2448900$ & $\mathrm{~V}$ & $(\mathrm{~b}-\mathrm{y})$ & $\mathrm{m}_{1}$ & $\mathrm{c}_{1}$ \\
\hline 28.6757 & 7.417 & .187 & .083 & .788 \\
& & & & \\
28.6796 & 7.456 & .184 & .088 & .762 \\
28.6812 & 7.469 & .186 & .089 & .761 \\
28.6857 & 7.473 & .195 & .070 & .775 \\
28.6874 & 7.471 & .179 & .091 & .752 \\
28.6913 & 7.446 & .164 & .090 & .757 \\
& & & & \\
28.6930 & 7.407 & .167 & .079 & .773 \\
28.6968 & 7.290 & .123 & .109 & .812 \\
28.6984 & 7.191 & .103 & .104 & .872 \\
28.7030 & 6.953 & .073 & .132 & .979 \\
28.7046 & 6.957 & .076 & .138 & .984 \\
& & & & \\
28.7092 & 7.042 & .100 & .132 & .970 \\
28.7108 & 7.091 & .112 & .129 & .940 \\
28.7155 & 7.212 & .149 & .102 & .887 \\
28.7172 & 7.252 & .147 & .113 & .855 \\
28.7213 & 7.327 & .168 & .114 & .834 \\
& & & & \\
28.7259 & 7.375 & .188 & .101 & .802 \\
28.7299 & 7.408 & .192 & .098 & .781 \\
28.7339 & 7.423 & .192 & .094 & .786 \\
28.7380 & 7.433 & .174 & .121 & .753 \\
28.7420 & 7.429 & .179 & .107 & .757 \\
28.7459 & 7.415 & .170 & .114 & .771 \\
28.7499 & 7.377 & .160 & .106 & .796 \\
28.7538 & 7.323 & .142 & .126 & .816 \\
\hline & & & & \\
\hline \hline
\end{tabular}


